\title{
Restoring Satisfactory Status in ThinPrep Pap Test Specimens With Too Few Squamous Cells and Containing Microscopic Red Blood Cells
}

Yijun Pang, M.D., Ph.D., ${ }^{\star}$ Brian Smola, C.T., Robert T. Pu, M.D., Ph.D., and Claire W. Michael, M.D.

Treatment of specimens that contain excessive blood can effectively reduce the unsatisfactory rate; however, a considerable number of unsatisfactory specimens remain. We evaluated the effectiveness of reprocessing unsatisfactory specimens that had too few squamous cells and contained microscopic red blood cells (TFSQRBC).

Out of the 688 unsatisfactory specimens at microscopic screening, 197 (28.63\%) were TFSQRBC that were reprocessed by treatment of glacial acetic acid (GAA). Red blood cells were observed clogging the pores in the filter of the ThinPrep device. After reprocessing, 129 (68.48\%) yielded a satisfactory diagnosis, which accounted for a reduction of the unsatisfactory rate by $18.25 \%$. In the restored satisfactory specimens, abnormal diagnoses of 1 high-grade squamous intraepithelial lesion (HSIL) (0.78\%), 3 atypical glandular cells (AGC) (2.33\%), and 13 atypical squamous cells of undetermined significance (ASCUS) (10.08\%) were made. The abnormal diagnoses in this group of patients were significantly higher than that in the general population screened.

Reprocessing unsatisfactory ThinPrep ${ }^{\mathbb{B}}$ (TP) specimens of $T F S Q R B C$ can reduce the unsatisfactory rate of the TP Pap test significantly and is a cost-effective measure. The initially unsatisfactory specimens are more likely to represent cases with an abnormal diagnosis, which also justifies the effort of reprocessing this group of specimens. Adjustment of the pore size on the ThinPrep filter device may reduce the interference of red blood cells. Diagn. Cytopathol. 2008;36:696-700. @ 2008 Wiley-Liss, Inc.

Key Words: Pap test; unsatisfactory; ThinPrep; reprocessing

Department of Pathology, University of Michigan, Ann Arbor, Michigan

*Correspondence to: Yijun Pang M.D., Ph.D., Department of Pathology, University of Michigan, 1500 E Medical Canter Drive, Ann Arbor, Michigan 48109. E-mail: ypang@umich.edu

Received 14 December 2007; Accepted 9 May 2008

DOI 10.1002/dc.20890

Published online in Wiley InterScience (www.interscience.wiley.com).
Unsatisfactory specimens exist in both conventional Pap smears and liquid-based Pap tests. ThinPrep ${ }^{\circledR}$ (TP) (Cytyc Corp., Marlborough, MA) is one of the liquid-based cervical cytology processing techniques. The unsatisfactory rate with TP ranges from $0.3 \%$ to $8.3 \%$ at various institutions. ${ }^{1-7}$ Compared with conventional Pap smears (CP), the frequency of unsatisfactory specimens with TP might be either more or less. ${ }^{1-5}$ The split specimen studies demonstrated an overall increase of unsatisfactory specimens with TP. ${ }^{8}$

The 2001 Bethesda criteria define that an adequate liquid-based preparation (LBP) should have an estimated minimum of 5000 well-visualized/well-preserved squamous cells. The most frequent causes of unsatisfactory status in TP specimens are too few squamous cells, followed by obscuration of red blood cells, white blood cells, and mucin..$^{9-15}$ Because TP is a filter-based processing technique, non-squamous cell particles, such as white and red blood cells (RBC) and mucin, compete with squamous cells to adhere on the filter, which results in too few squamous cells on the slide. Pretreatment of specimens containing grossly visible blood with glacial acetic acid (GAA) is effective in reducing the unsatisfactory rate. ${ }^{9,11,12,14,15}$ However, a subgroup of unsatisfactory specimen with too few squamous cells was identified with presence of microscopic red blood cells (TFSQRBC). In this study, we evaluated the effectiveness of reprocessing unsatisfactory TP specimens of TFSQRBC and the follow-up outcomes of these patients.

\section{Materials and Methods}

\section{Subjects}

A total of 45,418 consecutive TP specimens in the Department of Pathology, at the University of Michigan in the time period from September 1, 2005 to August 31, 
2006 were studied, including patients seen at the University of Michigan Health Care system, and community hospitals and clinics in the vicinity of Ann Arbor, Michigan. TP specimens were obtained by the clinicians and submitted to the Department of Pathology. College students were a noticeable portion in the population studied.

\section{Specimen Preparation}

The specimens were collected in PreservCyt ${ }^{\circledR}$ (Cytyc, MA). Before processing, the specimens were visually inspected. Those containing grossly visible blood were pretreated with GAA according the methods previously reported. ${ }^{13}$ ThinPrep slides of all specimens including the pretreated ones were prepared on a TP $3000^{\circledR}$ (Cytyc, MA).

\section{Identification of Unsatisfactory Specimens and Reprocessing}

Upon microscopic screening, the specimens were identified for reprocessing based on the following criteria: (1) unsatisfactory due to too few squamous cells and (2) the presence of intact red blood cells. The Bethesda System 2001 was used for the definition of satisfactory. To determine the unsatisfactory status, squamous cells were counted in 10-20 fields under $\times 400$ magnification across two diameters of the slide. An average less than 3 cells per field is considered to be too few squamous cells. The presence of any number of intact red blood cells was considered to fulfill the second criteria for the specimen to be reprocessed. The remaining portion of identified specimens was centrifuged at 2,500 $\mathrm{g}$ for $5 \mathrm{~min}$ and the supernatant was discarded. The cells were resuspended with gentle vortex in Cytolyt ${ }^{\circledR}$ that contained $10 \%$ GAA. One additional TP slide was made from the reprocessed specimen. The additional slide and the original slide were rescreened under microscopy for a diagnosis.

\section{Observation of RBC in the ThinPrep Device Filter}

The process was interrupted immediately before the cells were delivered to glass slides. The filter with the collected cells was taken off, fixed in 95\% ethanol, and processed with Pap stain. Then the filter was adhered on a glass slide and coversliped with mount gel.

\section{Data Analysis}

Patients' age, referring physician, information of last menstrual period, and outcome of the reprocessed Pap test were collected. Fisher's test were performed to test the significance of the reprocessing efforts and to identify factors potentially causing the unsatisfactory diagnosis. Twotailed $P$-values were calculated to assess the statistical significance. The $P$-value $<0.05$ was considered to be statistically significant.

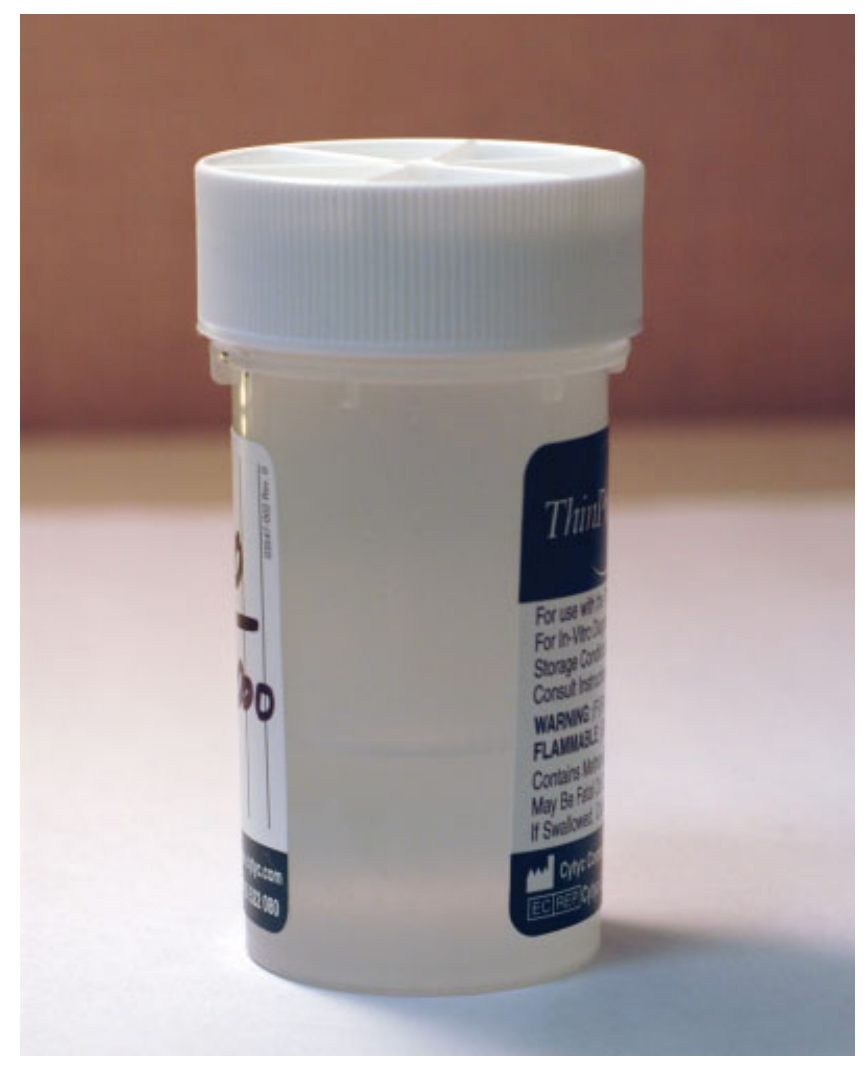

Fig. C-1. Hypocellular specimen containing microscopic red blood cells is not grossly bloody.

\section{Results}

We identified 688 unsatisfactory specimens by microscopic screening. Among all initially unsatisfactory specimens, $197(28.63 \%)$ cases met the criteria for reprocessing. Grossly, no visible blood was present in those specimens. Hence, they had not been pretreated (Fig. C-1). After reprocessing, red blood cells were lysed and the number of squamous cells and endocervical cells increased (Fig. C-2). A satisfactory status was restored in $129(65.48 \%)$ specimens. Restored satisfactory status in the reprocessed specimens resulted in the current unsatisfactory rate of $1.23 \%$, a reduction of $18.25 \%$ from that of $1.51 \%$, if the reprocessing had not been conducted. The decrease is statistically significant $(P<0.001)$.

Abnormal diagnoses were made on 17 (13.18\%) specimens of restored adequacy with a distribution of 1 $(0.78 \%)$ high-grade squamous intraepithelial lesion (HSIL) (Fig. C-3), 3 (2.33\%) atypical glandular cells (AGC), and $13(10.08 \%)$ atypical squamous cells of undetermined significance (ASCUS). Comparison of abnormal findings between the reprocessed group and the general population screened is presented in Table I. The abnormal specimens exist more frequently in the reprocessed group $(10.08 \%)$ than that in the general population screened in the year 2005-2006 $(6.91 \%)(P=0.0088)$. The diagnoses 
PANG ET AL.

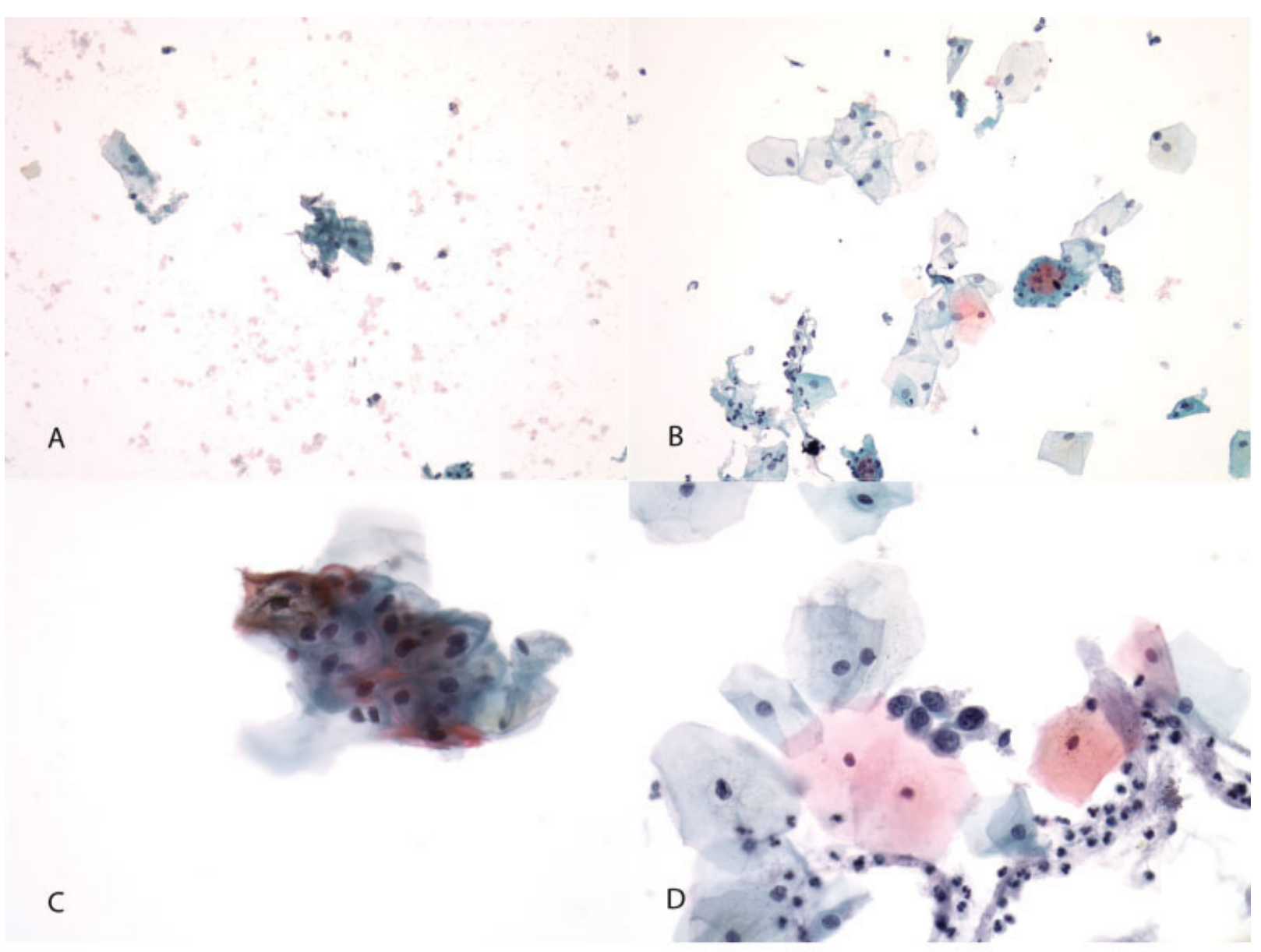

Fig. C-2

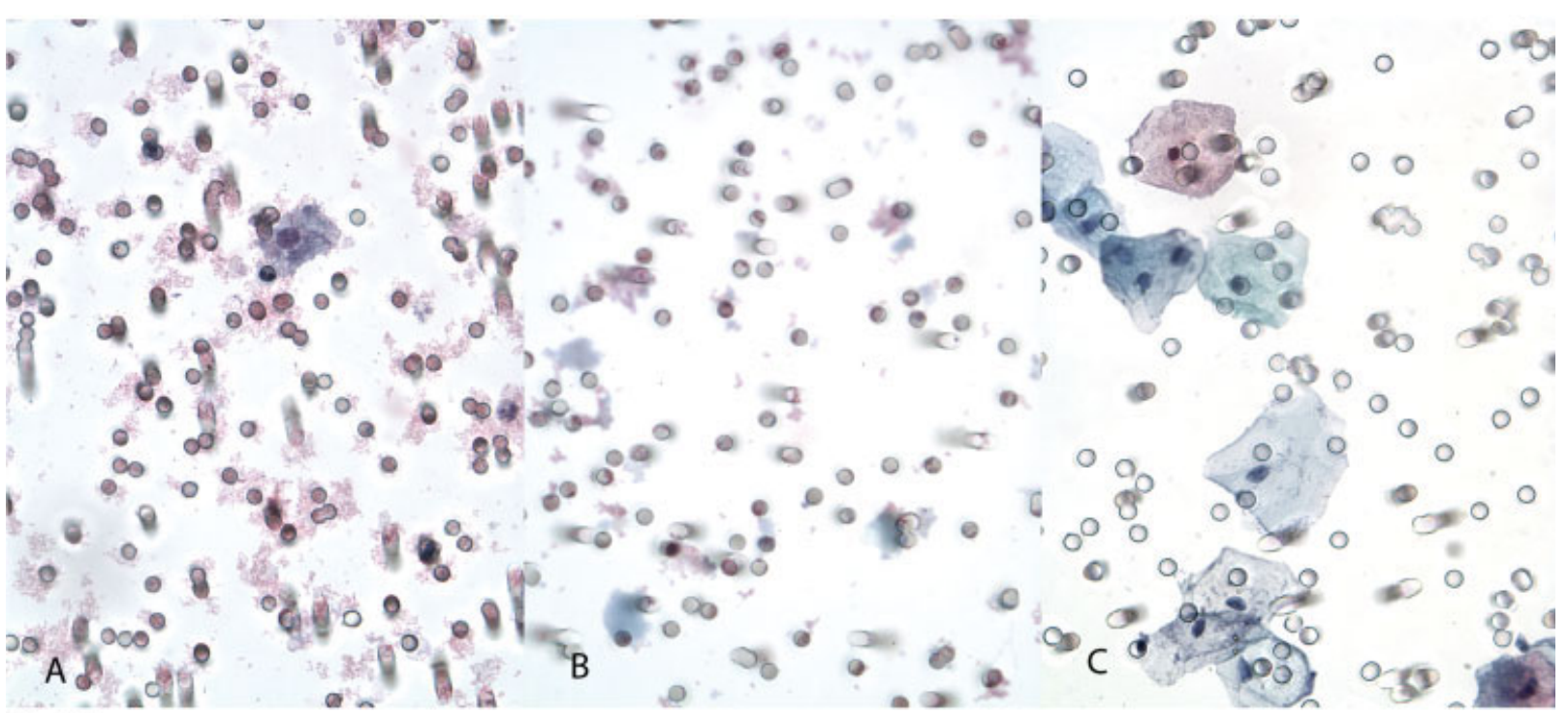

Fig. C-3

Figs. C-2-C-3. Fig. C-2. A: Hypocellular specimen containing intact red blood cells, $\times 200$. B: Restored cellularity after reprocessing. Debris of lysed red blood cells present. A negative diagnosis was made based on this specimen, $\times 200$. C: Diagnosis of low grade squamous intraepithelial lesion was made after reprocessing, $\times 400$. D: Diagnosis of high-grade squamous intraepithelial lesion was made after reprocessing, $\times 400$. Fig. C-3. A: The pores in the filter were clogged by red blood cells in the ThinPrep specimen. B: Lysed RBC debris in the pores of the filter. C: No interference with the filter in specimen containing no RBC. 
Table I. Abnormal Findings in Reprocessed Specimens and the General Population

\begin{tabular}{lcrrr}
\hline Category & HSIL & ASCUS & \multicolumn{1}{c}{ AGC } & Overall \\
\hline Reprocessed & $0.78 \%$ & $10.08 \%$ & $2.33 \%$ & $13.18 \%$ \\
General & $0.36 \%$ & $3.62 \%$ & $0.11 \%$ & $6.91 \%$ \\
$P$-value & 0.9583 & 0.0003 & $<0.0001$ & 0.0088 \\
\hline
\end{tabular}

of ASCUS and AGC are both significantly more frequent among the specimens with restored satisfactory status (Table I).

In patients with reprocessed specimens, the age ranged from 14 to 84 years with a median age of 38 years. To identify the possible factors that may be significant in causing unsatisfactory status, we looked into the average last menstrual period time (LMP), post menopause status, and possible association with the collector of the specimen.

We considered patients aged 55 years or older as post menopausal. Of all the patients with reprocessed specimens, $10.65 \%$ (21/197) were post menopausal. Post menopausal patients were significantly more frequent in the group in which unsatisfactory status could not be restored by reprocessing $(17.64 \%, 12 / 68)$, than in the group that unsatisfactory status was restored $(6.98 \%, 9 / 129)$ (Fisher's exact test $P=0.028$ ).

The last menstrual period (LMP) information was available in 119 patients with regular menstrual cycles. The mean time period from LMP to the specimen collecting time (SCT) was 9.7 days, and the median was 7 days with a range of 1-30 days.

The information regarding specimen collectors was available for 150 reprocessed specimens, associated to 107 clinics. More than 1/3 (66/150) of the reprocessed specimens were collected in 23 clinics that contributed 2 or more specimens, and one of the clinics had 5 specimens in this group, suggesting that these clinics had higher tendency to provide suboptimal samples.

Red blood cells in the ThinPrep specimen were observed clogging the pores of the ThinPrep device filter. The clogging was reduced in a processed specimen and debris of red blood cells was present in the pores of the filter. No clogging was observed in specimen containing no red blood cells (Fig. C-3).

\section{Discussion}

TFSQRBC is a subgroup of specimens that are initially unsatisfactory and would have not been pretreated with GAA, because the RBC was not grossly visible. During the time period of this study, all the pretreated specimens ( $n=254$ ) were satisfactory. The TFSQRBC accounted for more than a quarter $(28.63 \%)$ of all unsatisfactory specimens. The rate of restored satisfactory status $(65.48 \%)$ in this specific group was similar to that in the reprocessed unsatisfactory specimens with gross blood as previously reported. The reduction $(18.25 \%)$ of the unsatisfactory rate was significant. It is evident that the presence of red blood cells, even only microscopic, can be a significant factor to cause unsatisfactory TP preparations. Recognizing and reprocessing this group of unsatisfactory specimens with GAA is an effective procedure to reduce the unsatisfactory rate. It has been reported that up to $26 \%$ of the patients with an unsatisfactory Pap test specimen have an abnormal follow-up diagnosis. ${ }^{16} \mathrm{~A}$ repeat Pap test is required for patients with an unsatisfactory specimen, which demand additional clinic visits. Even worse, many of the patients may be lost in follow ups and miss the opportunity for prompt detection and intervention of a cervical lesion. ${ }^{11,16}$ Our study showed that the frequency of abnormal findings is significantly higher in patients with an unsatisfactory Pap specimen than that of the overall screened population (Table I). The efforts to restore the satisfactory status of this group of specimens are justified. Although reprocessing these unsatisfactory specimens may increase cost at $160 \%,{ }^{11}$ comparing the increase in reprocessing cost to the cost if the patient had gone back to the clinic for a repeat Pap test specimen, reprocessing this subgroup of specimens is a financially efficient measure. Most significantly, the integrity of the Pap test efficiency was restored and the patients retained the opportunity to be tested properly.

Among the factors that associated or contributing to the unsatisfactory status of ThinPrep specimen were found that the SCT relating to menstrual period is significant. The median SCT was 7 days post LMP indicating that the majority of these specimens were collected within 7 days of the LMP. Previous studies suggested that specimens collected mid-cycle of the menstrual period are optimal for both cytology and HPV tests and the specimens collected in day 4 to 6 of LMP were most frequently unsatisfactory. ${ }^{17,18}$ This finding should catch the attention of clinician colleagues. It is encouraged that the collection of a Pap test specimen should be at a time close to the middle of the menstrual cycle. Post menopausal status was significant, because atrophy of squamous mucosa is frequent. Consequently, less squamous cells are obtained in the specimen because the squamous epithelium is thinner and the squamous cells are smaller and more cohesive. It is also true that the tendency of bleeding exists when the atrophic cervices are sampled for Pap test. These statements are supported by our data that the Pap test specimens of post menopausal patients are more likely to remain unsatisfactory after being reprocessed. Correcting the atrophy by topical estrogen therapy is recommended for this group of patients before a repeat Pap test. Although the Pap specimen collection seems to be a simple, standardized procedure and most of the involved clinicians had been educated, the quality of the specimen 
is after all operator-dependent. Review of the specimen collecting techniques among the small number of collectors who generated unsatisfactory Pap test specimens may be a useful approach to reduce the number of unsatisfactory specimens.

Obscuration by blood and its consequence of too few squamous cells are among the major causes of unsatisfactory. Bentz et al. demonstrated that coexisting red blood cells compete with squamous cells to be retrieved on the filter that transfer the cells to the slides. ${ }^{11}$ Our observation of ThinPrep device filters with specimens provided direct visual evidence that intact RBC interfere with the processing of ThinPrep specimen through clogging the pores of the ThinPrep device filter. The pore size of the filter is estimated about $10 \mathrm{~mm}$ in diameter, approximate the diameter of the red and white blood cells. If the pore size is enlarged to a size that allows RBC to pass, the interference may be reduced.

In summary, reprocessing unsatisfactory ThinPrep specimens that contain microscopic red blood cells is a practical and cost-effective procedure, resulting in significant reduction of unsatisfactory rate. A patient with such a specimen is at higher risk of harboring a cervical lesion. Post menopausal status and the specimen collecting day close to LMP may be causes for the unsatisfactory specimen. Further education to the specimen collectors may improve the quality of specimen.

\section{References}

1. Grace A, McBrearty P, Troost S, Thornhill M, Kay E, Leader M. Comparative study: Conventional cervical and ThinPrep Pap tests in a routine clinical setting. Cytopathology 2002;13:200-205.

2. Roberts JM, Gurley AM, Thurloe JK, Bowditch R, Laverty CR. Evaluation of the ThinPrep Pap test as an adjunct to the conventional Pap smear. Med J Aust 1997;167:466-469.

3. Papillo JL, Zarka MA, St. John TL. Evaluation of the ThinPrep Pap test in clinical practice. A seven-month, 16,314-case experience in northern Vermont. Acta Cytol 1998;42:203-208.
4. Yeoh GP, Chan KW, Lauder I, Lam MB. Evaluation of the ThinPrep Papanicolaou test in clinical practice: 6-Month study of 16,541 cases with histological correlation in 220 cases. Hong Kong Med J 1999;5:233-239.

5. Nance KV. Evolution of Pap testing at a community hospital: A ten year experience. Diagn Cytopathol 2007;35:148-153.

6. Bentz JS. Liquid-based cytology for cervical cancer screening. Expert Rev Mol Diagn 2005;5:857-871.

7. Tuncer ZS, Basaran M, Sezgin Y, Firat P, Mocan Kuzey G. Clinical results of a split sample liquid-based cytology (ThinPrep) study of 4,322 patients in a Turkish institution. Eur $\mathrm{J}$ Gynaecol Oncol 2005;26:646-648.

8. Siddiqui MT, Gokaslan ST, Saboorian MH, Ashfaq R. Split sample comparison of ThinPrep and conventional smears in endoscopic retrograde cholangiopancreatography-guided pancreatic fine-needle aspirations. Diagn Cytopathol 2005;32:70-75.

9. Song LH, Goh ES, Phang LC, Poh WT, Tay SK. Technical aspect of ThinPrep. Singapore Med J 2000;41:575-578.

10. Agoff SN, Dean T, Nixon BK, Ingalls-Severn K, Rinker L, Grieco VS. The efficacy of reprocessing unsatisfactory cervicovaginal ThinPrep specimens with and without glacial acetic acid: Effect on Hybrid Capture II human papillomavirus testing and clinical followup. Am J Clin Pathol 2002;118:727-732.

11. Bentz JS, Rowe LR, Gopez EV, Marshall CJ. The unsatisfactory ThinPrep Pap Test: Missed opportunity for disease detection? Am J Clin Pathol 2002;117:457-463.

12. Islam S, West AM, Saboorian MH, Ashfaq R. Reprocessing unsatisfactory ThinPrep Papanicolaou test specimens increases sample adequacy and detection of significant cervicovaginal lesions. Cancer 2004;102:67-73.

13. Rowe LR, Bentz JS. A simple method to determine the need for glacial acetic acid treatment of bloody ThinPrep Pap tests before slide processing. Diagn Cytopathol 2004;31:321-325.

14. Dalton P, MacDonald S, Boerner S. Acetic acid recovery of gynecologic liquid-based samples of apparent low squamous cellularity. Acta Cytol 2006;50:136-140.

15. Haack LA, O'Brien D, Selvaggi SM. Protocol for the processing of bloody cervical specimens: glacial acetic acid and the ThinPrep Pap Test. Diagn Cytopathol 2006;34:210-213.

16. Ransdell JS, Davey DD, Zaleski S. Clinicopathologic correlation of the unsatisfactory Papanicolaou smear. Cancer 1997;81:139-143.

17. Vooijs GP, van der Graaf Y, Elias AG. Cellular composition of cervical smears in relation to the day of the menstrual cycle and the method of contraception. Acta Cytol 1987;31:417-426.

18. Sherman ME, Carreon JD, Schiffman M. Performance of cytology and human papillomavirus testing in relation to the menstrual cycle. Br J Cancer 2006;94:1690-1696. 